International Journal on Applied Bioengineering, Vol. 5, No.1, Jaunary 2011

\title{
PERFORMANCE EVALUATION OF EEG AND MEG BASED SLEEP ONSET DETECTION USING MEAN COMPARISON TEST
}

\author{
Sivaramakrishnan $R^{1}$, Ramanathan P.K ${ }^{2}$, Vidhya $S^{3}$, Arun $C^{4}$ \\ 1,2,3 Department of Biomedical Engineering, SSN College of Engineering, (TN), India, \\ ${ }^{4}$ Department of Electronics and Communication Engineering, Rajalakshmi Engineering college, (TN), India \\ Email: ${ }^{1}$ raaju.shiv@gmail.com
}

\begin{abstract}
The objective of this project is to detect the onset of drowsiness/sleep by an analysis of the characteristic features using EEG and MEG waveforms corresponding to the "Awake" and "Drowsy" states using MCT (Mean Comparison Test) approach. Drowsiness refers to an abnormally sleepy feeling, especially at a time when the subject is required to be awake and alert. Such momentary feelings of drowsiness may lead to catastrophic errors of judgment. The proposed system detects the condition of drowsiness by acquiring the physiological signals such as EEG and MEG; the time and frequency domain characteristics of those signals change depending upon the level of alertness. The acquired EEG and MEG signals are generally distorted due to the addition of noise from various sources as well as by parasitic biological artifacts. So, filtering plays a vital role in the preprocessing of the data. The useful features giving information on awake, drowsy and sleep states of the subject can be extracted by implementing wavelet decomposition and windowing technique. These extracted features are then supplied as inputs to the classifier, which classifies them into awake and drowsy state by following a set of rules and procedures assigned initially.
\end{abstract}

Keywords: EEG, MEG, MCT, ROC

\section{INTRODUCTION}

Drowsiness refers to a feeling abnormally sleepy during the day. Drowsiness in people may lead to falling asleep in inappropriate situations or at inappropriate times such as during driving. Also, drowsiness is most characterized in people with a monotonous work conditions. These momentary lapses may lead to catastrophic errors. There are several drowsiness detection systems available currently worldwide which are extensively used by drivers and night workers. Traditional drowsiness detection systems mainly work on principle of digital image processing techniques, where the facial image of the user is captured and processed for fatigue level estimation. Also, tilt and IR sensors aid in tracking the closure of eyes and facial rigidity. Even the bio-signals like PPG, $E O G, E C G$ and EEG have partially ventured in this avenue as the physiological parameters change with respect to the degree of alertness like the slight decrease in respiration rate and body temperature. But these systems lag in the time taken for the detection and response. We require a suitable algorithm which could help us in detecting the onset of sleep even before it is expressed facially. Hence, the EEG (electroencephalography) signals can best suit the requirement.
Electroencephalography is a powerful non-invasive method for medical diagnosis, which can provide high temporal and spatial resolution in milli-seconds that directly reflects the dynamics of the generating cell assemblies. The recordings are the summation of volume conductor fields produced by millions of interconnecting neurones. The architecture of the brain is not uniform but varies with different locations. Thus the EEG can vary depending on the location from which it is acquired. During the transition from awake state to Stage I sleep state (drowsiness), there is a drastic change in the EEG rhythms. The power of alpha is expected to increase drastically with a decrease in the beta power at the onset. This feature of the EEG signals is extracted out to classify the awake and drowsy states and to detect the onset of sleep.

Magnetoencephalography is an imaging technique (contact-less) used to measure the magnetic fields produced by the electrical activity in the brain via extremely sensitive devices superconducting quantum interference devices (SQUIDs) [1]. This technique has very high sensitivity and has higher spatial resolution than EEG. This property of MEG is established to localize the area which produces high accuracy for sleep signal reception and feedback. 


\section{METHODOLOGY}

\section{A. Data Acquisition}

The experiment was conducted at the MEG laboratory, IGCAR (Kalpakkam, India). The EEG signals were acquired from 3 subjects (1 female and 2 male) of age group 20-30 years. Five trials of duration of about 30 minutes each were taken for each subject. The recordings were performed using a 64-channel NeuroScan (EEG) System by placing an electrode cap on the subjects head. This system uses a cap consisting of 64 channels and EOG and EKG electrodes. The signals were acquired at a sampling rate of $1000 \mathrm{~Hz}$, digitized with a resolution of 24 bits and were recorded continuously applying band-pass filter from 0 to $200 \mathrm{~Hz}$ and a $50 \mathrm{~Hz}$ notch filter.

The MEG signals were mainly acquired from 4 electrodes placed over occipital lobe [7] from 3 subjects (1 male and 2 female) of the age group 20-30 years. Three trials of duration 30 minutes each were taken for each subject. The MEG Acquisition System consisted of 4 channels with the SQUID sensors dipped in liquid Helium at a low temperature of $4.2 \mathrm{~K}$ placed in a double walled vacuum insulated liquid helium cryostat. The MEG signals were acquired with a sampling frequency of $1000 \mathrm{~Hz}$, digitized with a resolution of 24 bits and were recorded continuously after applying a band-pass filter of $0-100 \mathrm{~Hz}$ and a 50 $\mathrm{Hz}$ notch filter; the data was stored for further processing and analysis. For MEG data acquisition, the subject was instructed to remove all magnetic or metallic materials worn by him before entering into the magnetically shielded room in order to avoid spurious artifacts.

\section{B. Pre-processing}

The basic pre-processing of the data involves filtering of the acquired signal and artifact removal. It is mainly done to improve the signal-to-noise ratio (SNR) of the recorded signal. The sampling frequency of the recorded EEG and MEG signals is $1000 \mathrm{~Hz}$. These recorded signals are then filtered using an FIR band pass filter with cut-off frequencies $2 \mathrm{~Hz}$ to $30 \mathrm{~Hz}$ to remove base-line drift, power-line interference and movement artifacts.

The spectrum showing the raw EEG signal recorded from NeuroScan for a period of 1 second and the spectrum of the raw EEG signal are in Fig 1-4 shown below:

\section{Resampling}

For proper decomposition of the EEG and MEG signals using wavelet decomposition technique, a sampling rate of 64 samples per second is required. For this reason, the above filtered signals are down sampled from 1000 samples per second to 64 samples per second. Feature extraction is the key tool used in the EEG data processing and analysis. The feature extraction refers to a process of identifying the main and valuable parameters from the EEG/MEG signal waveforms using both the time domain and the frequency domain analysis. It reduces the dimensionality in the classification algorithm by selecting the required parameters of the EEG and MEG

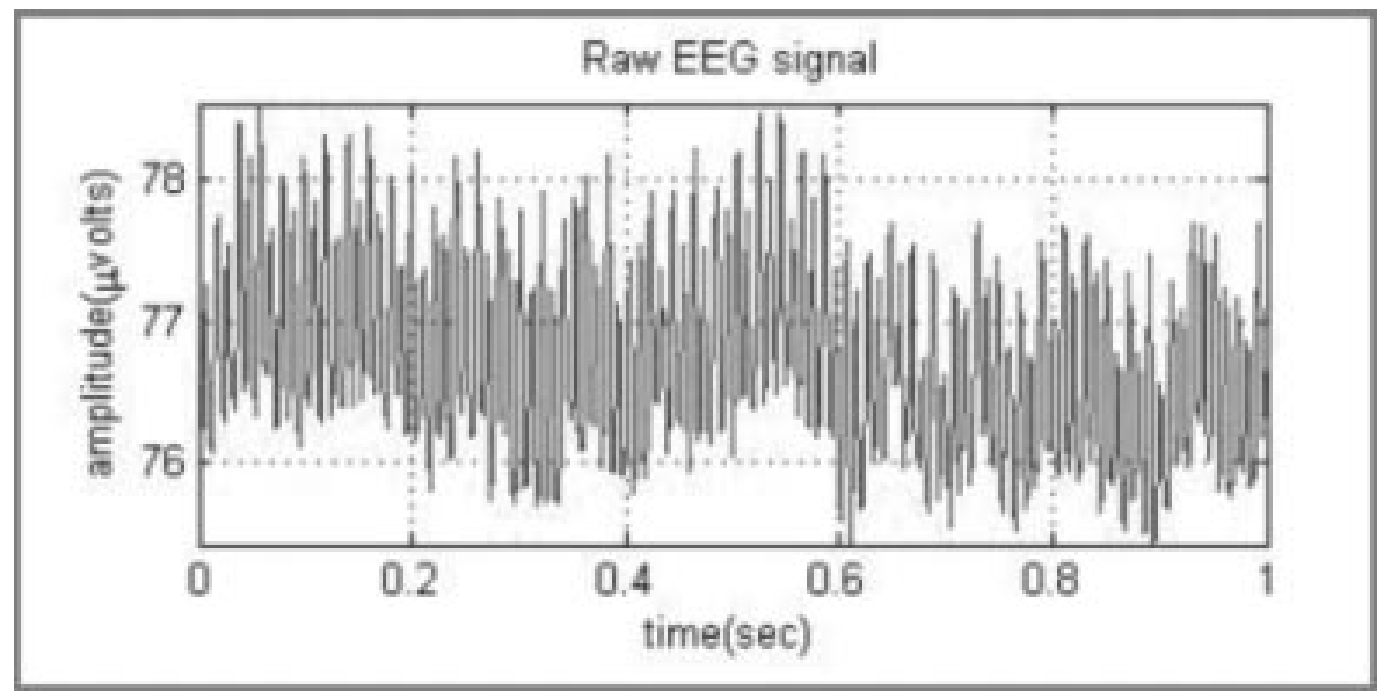

Fig. 1. Raw EEG signal recorded from NeuroScan (1sec) 


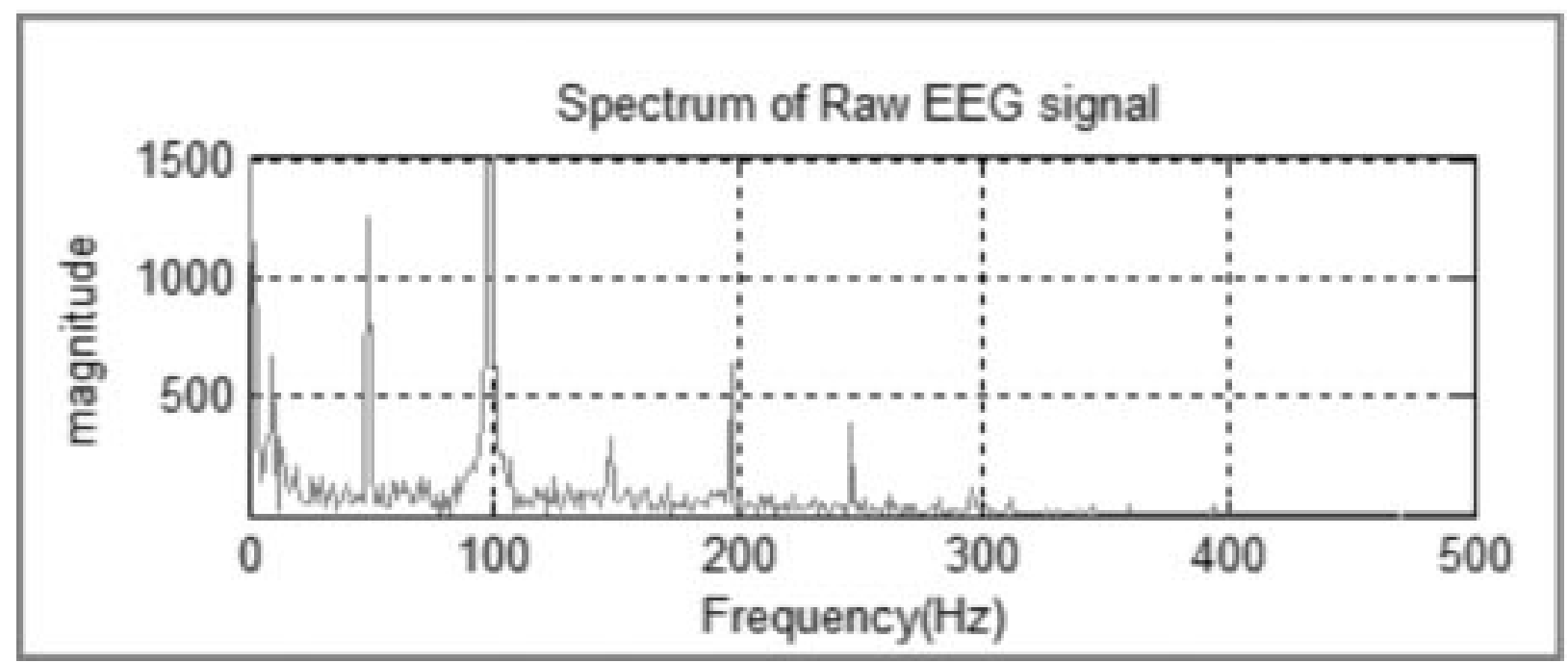

Fig. 2. Spectrum of Raw EEG signal

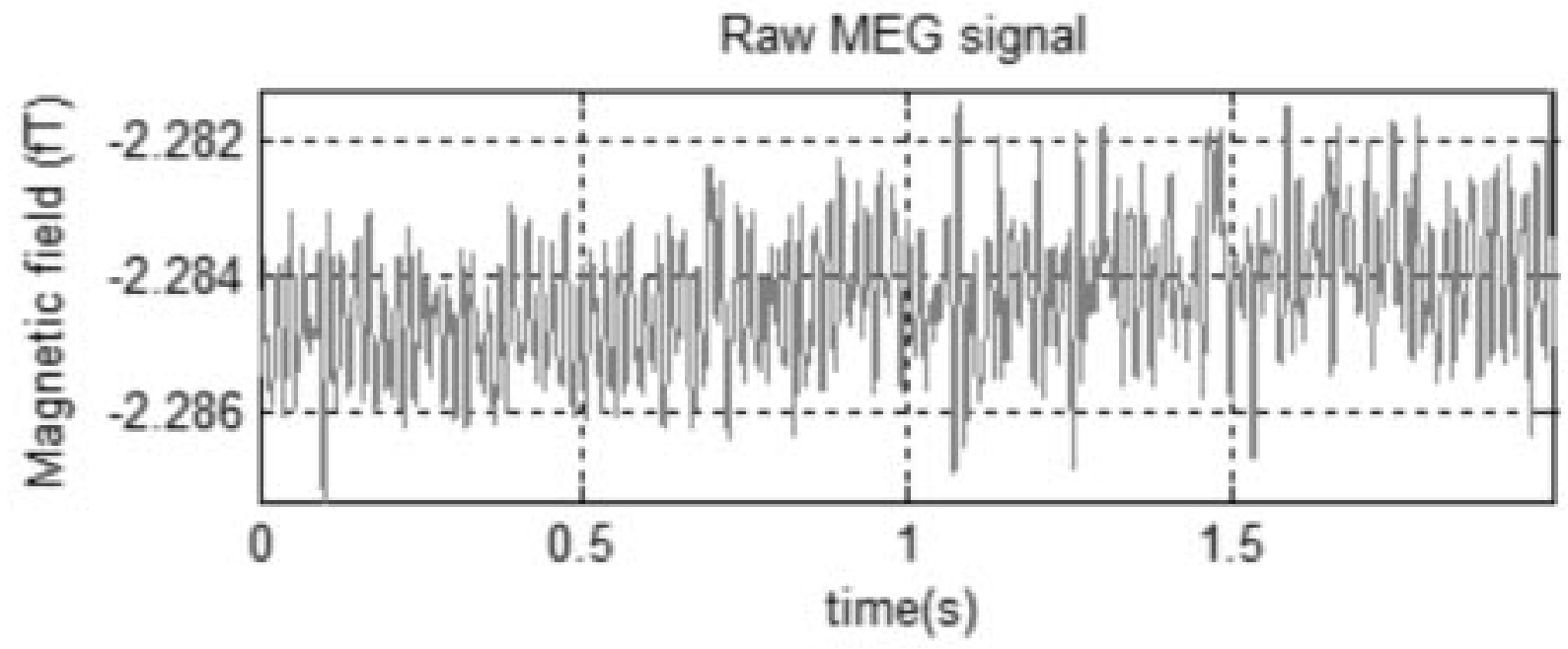

Fig. 3. Raw MEG signal recorded from the MEG equipment (2 sec)

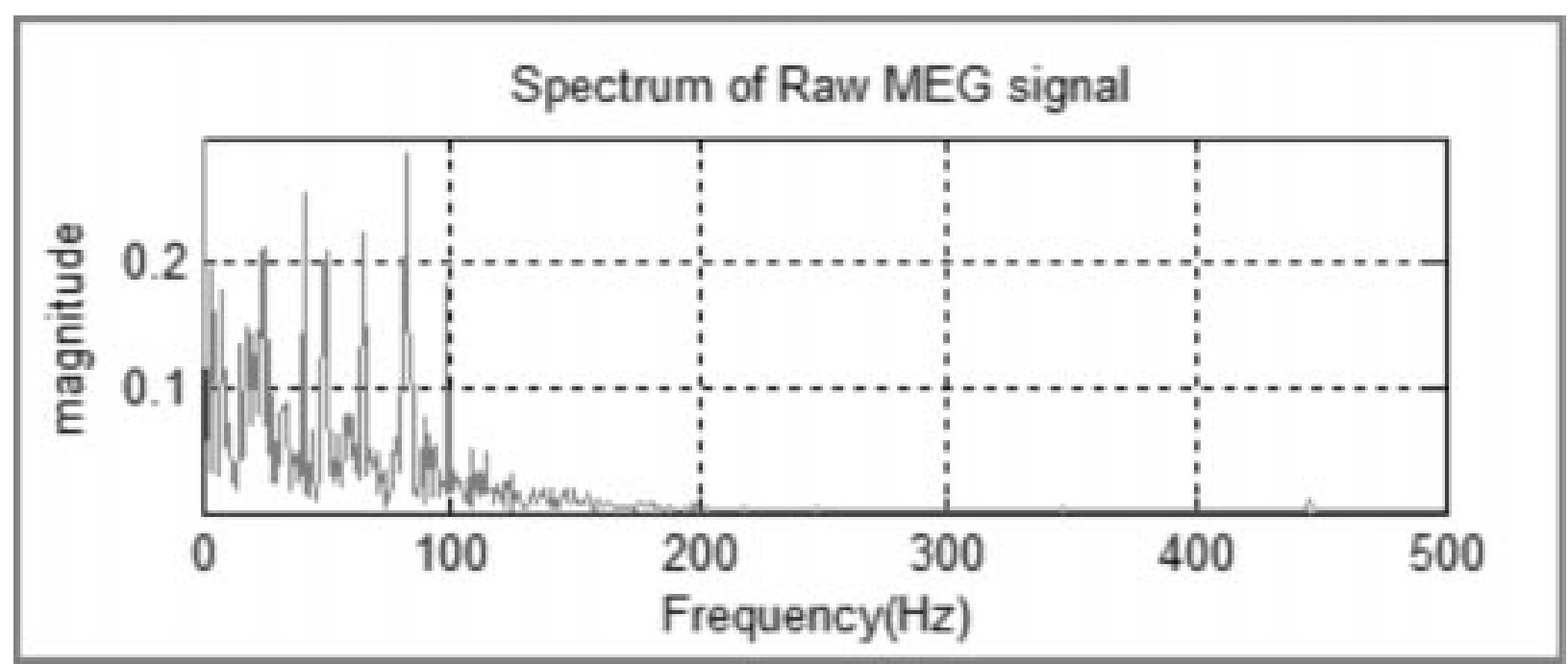

Fig. 4. Spectrum of Raw MEG signal 


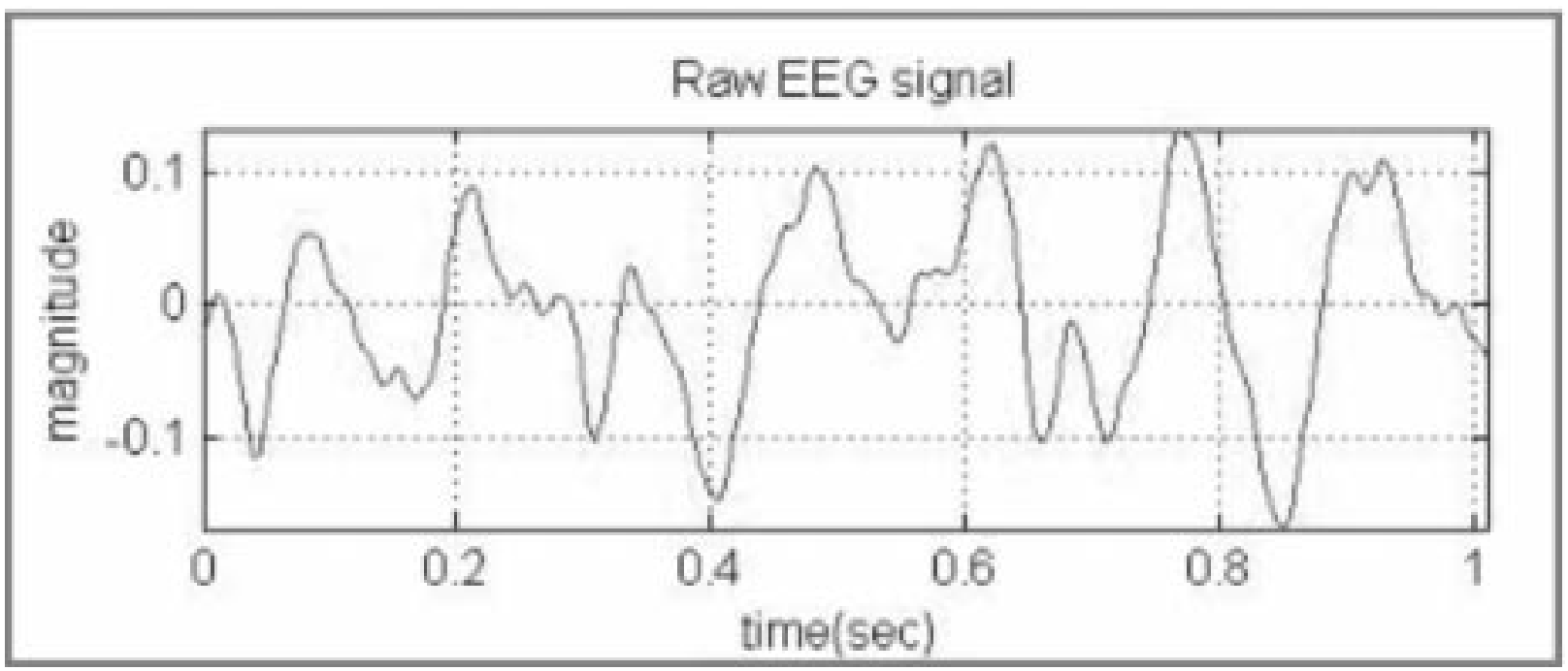

Fig. 5. Filtered EEG signal

\section{FFT of Filered EEG}

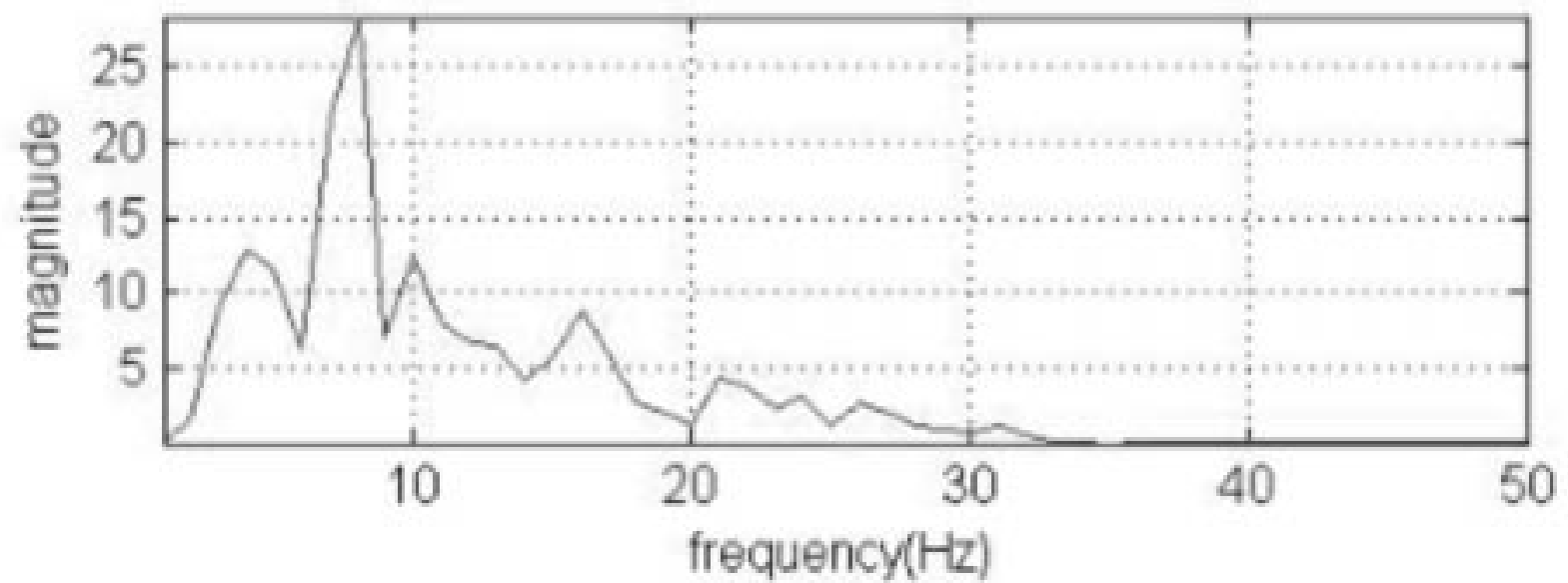

Fig. 6. Spectrum of Filtered EEG signal

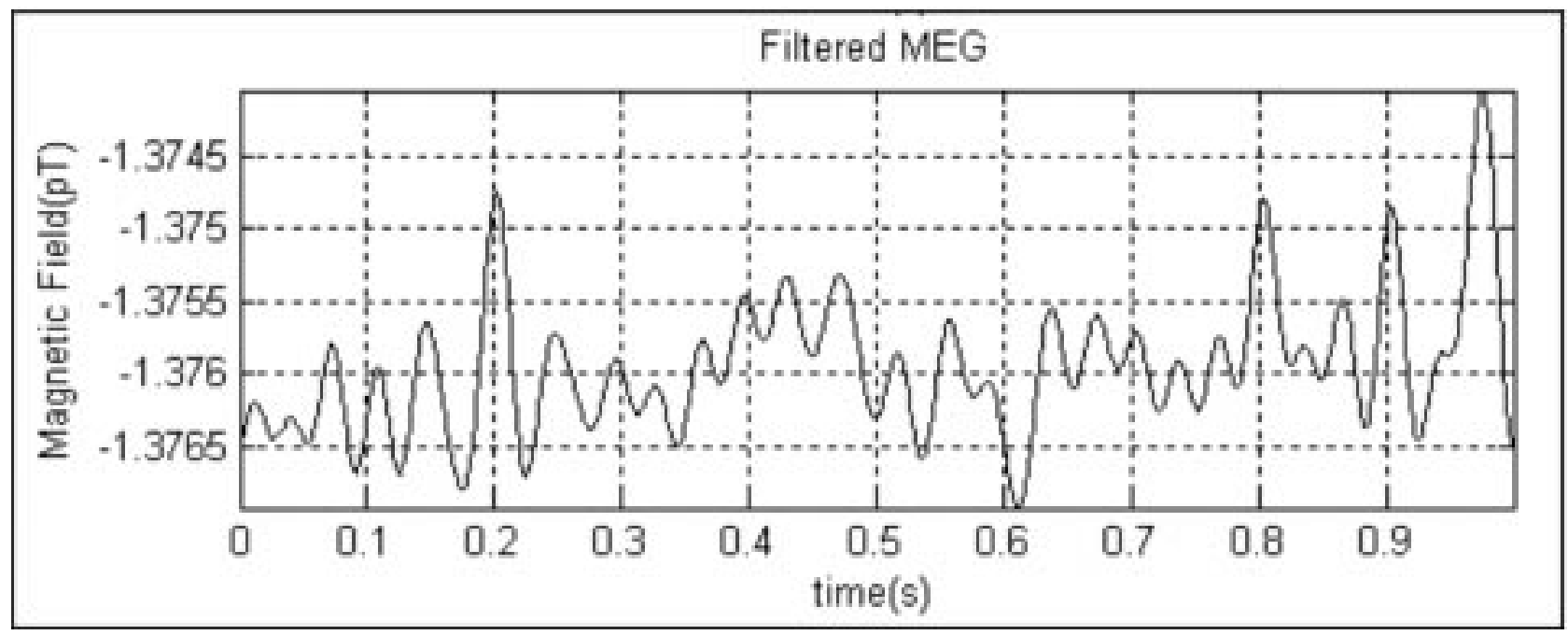

Fig. 7. Filtered MEG signal 


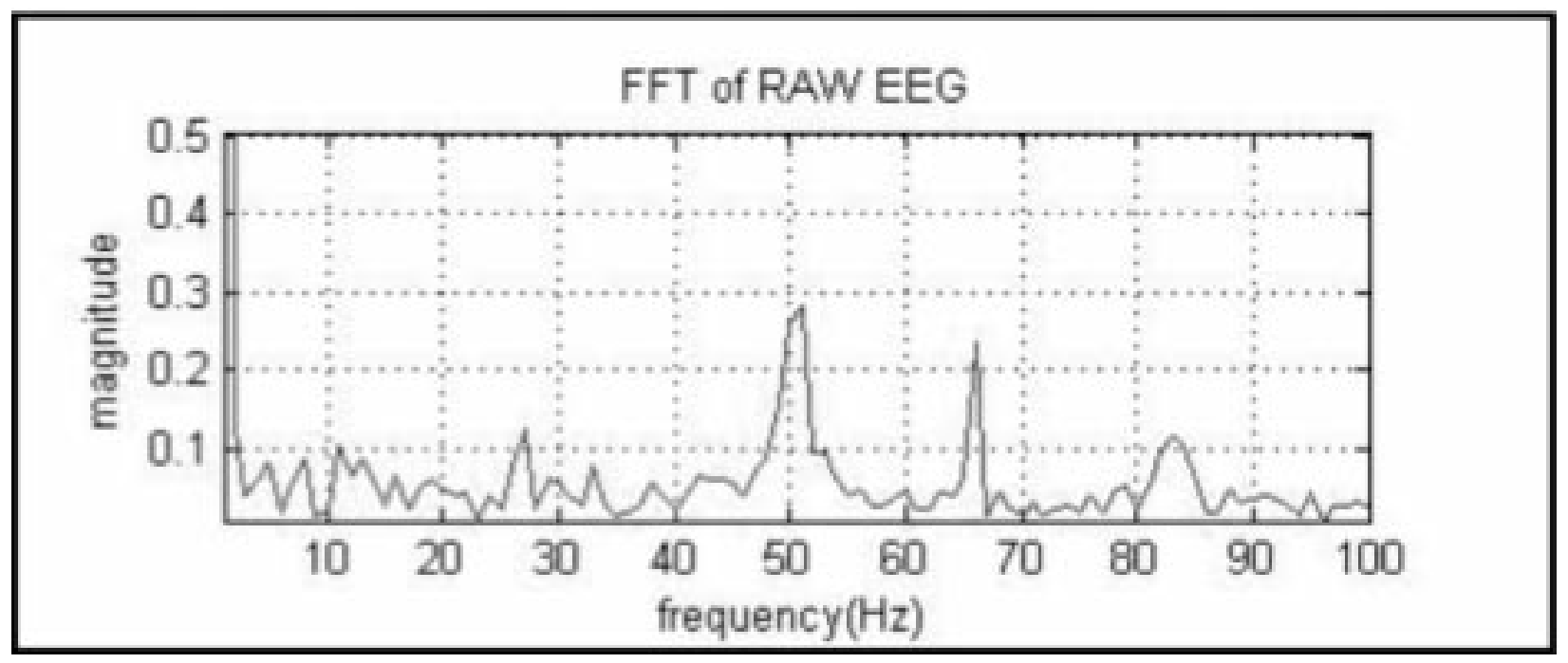

Fig 8: Spectrum of Filtered MEG signal

signals, which play a vital role in the classification of the state of the subject into "Awake" or "Drowsy" categories as shown in figure 5-8.

\section{FEATURE EXTRACTION}

\section{A. Segmentation using Windowing}

Due to the highly non-stationary nature of the EEG/MEG signals, the feature extraction algorithms are applied to segment the signal with different window sizes and overlap times. This analysis is done for different lengths of the windows, namely, 5 seconds window with 2 seconds overlap, 10 seconds window with 2 seconds overlap, and 20 seconds window with 5 seconds overlap.

\section{B. Wavelet Decomposition}

The segmented signals are then decomposed into beta, alpha, theta and delta bands by applying wavelet ( $\mathrm{db} 5$ ) and level 3 decomposition techniques. The original signal is band limited to $0-32 \mathrm{~Hz}$. [6] Thus, detailed coefficients of first level decomposition (CD1), when reconstructed correspond to the Beta band as shown in figure 9. Similarly, the cD2 and CD3 correspond to Alpha and Theta band, respectively. Also, the third level approximate coefficients (cA3) correspond to Delta band. The spectrum of decomposed EEG signal is shown in Fig.10
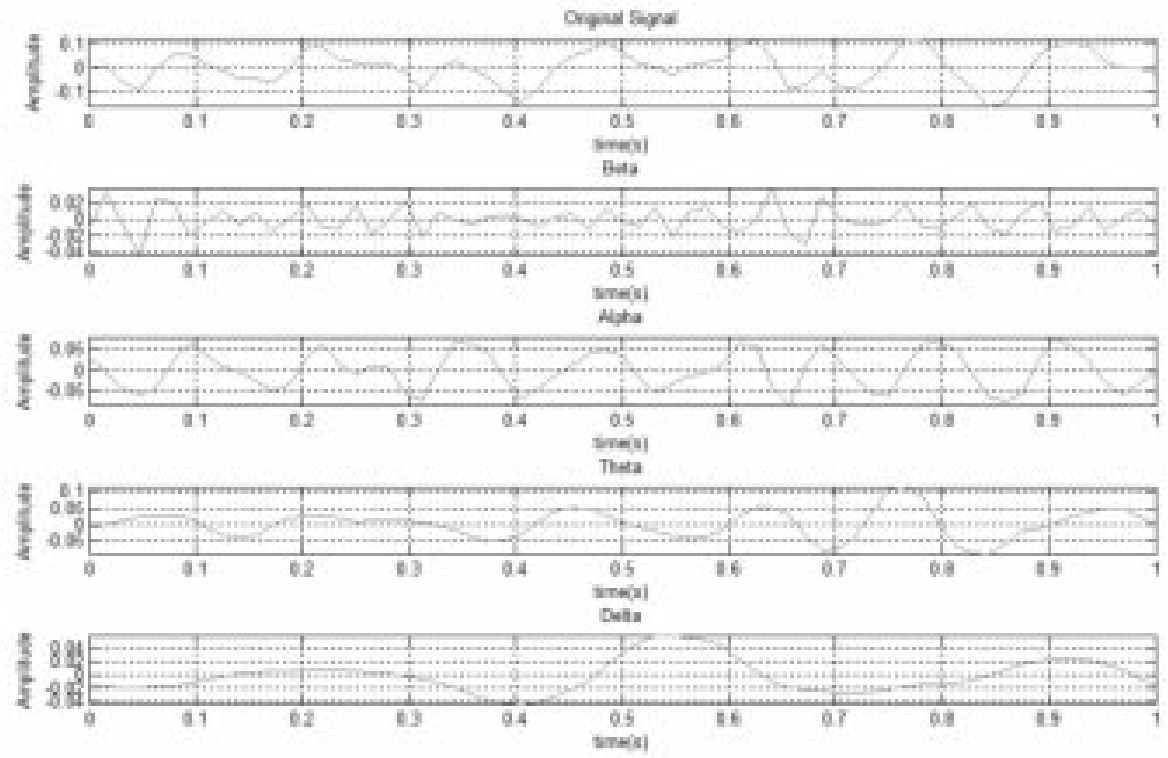

Fig 9. Decomposed EEG signals 


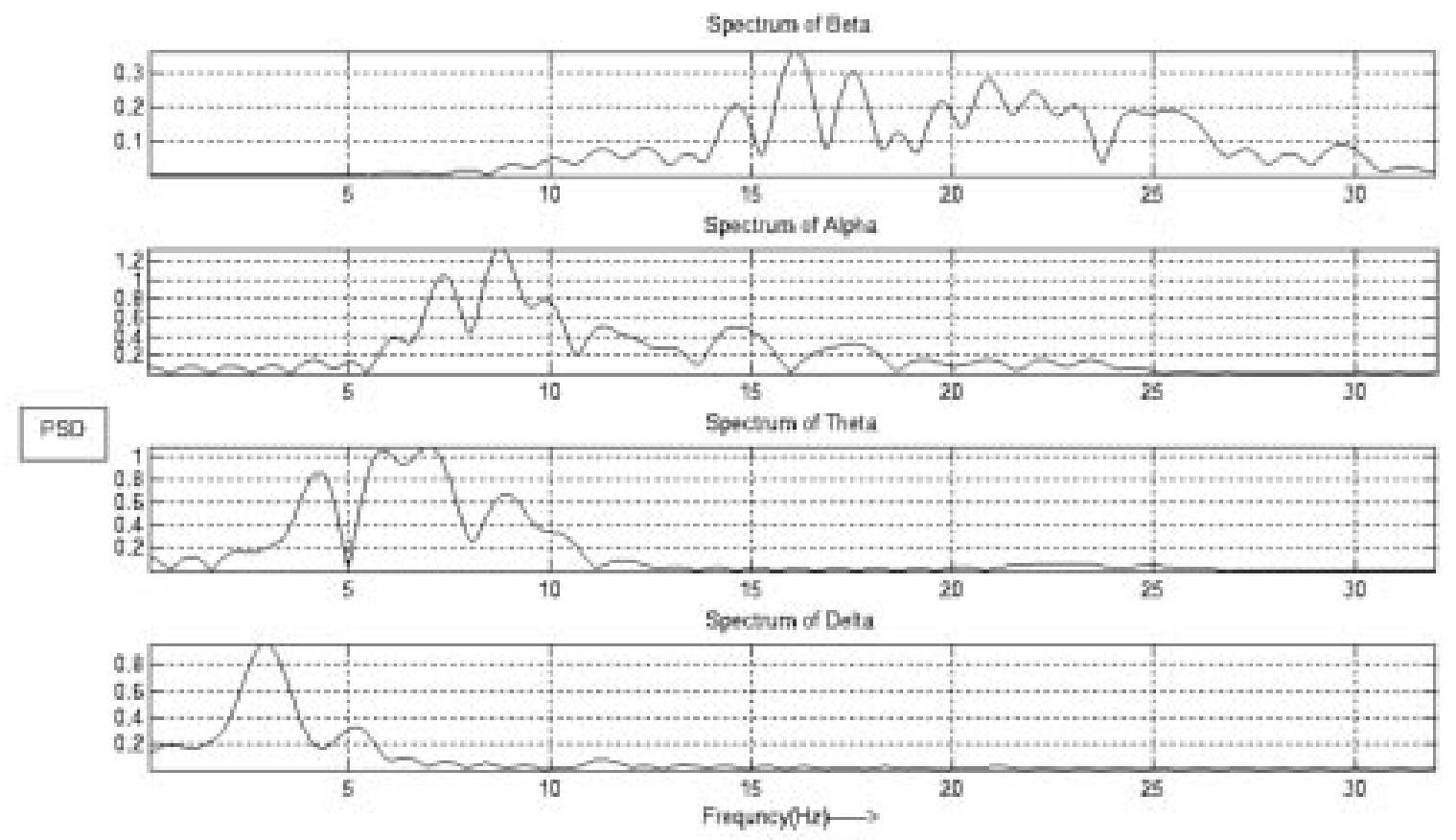

Fig 10. Spectrum of Decomposed EEG signals

\section{Relative Power Estimation}

The average power in each band for the decomposed EEG and MEG signals is estimated to calculate the relative power of the band, as given below:

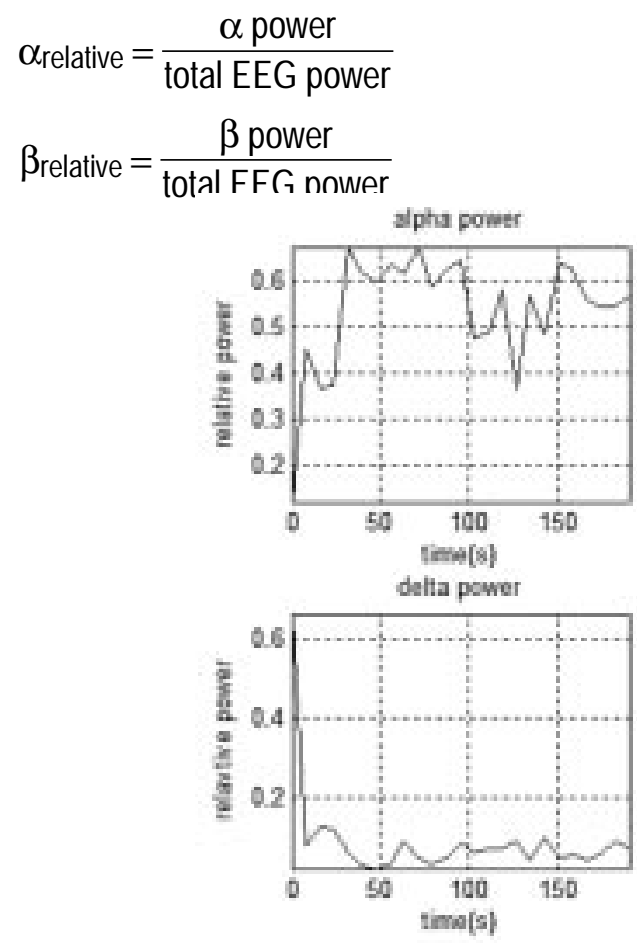

$$
\begin{aligned}
& \theta_{\text {relative }}=\frac{\theta \text { power }}{\text { total EEG power }} \\
& \delta_{\text {relative }}=\frac{\delta \text { power }}{\text { total EEG power }}
\end{aligned}
$$

where, $\alpha, \beta, \theta$, and $\delta$ denotes alpha, beta, theta and delta bands respectively.

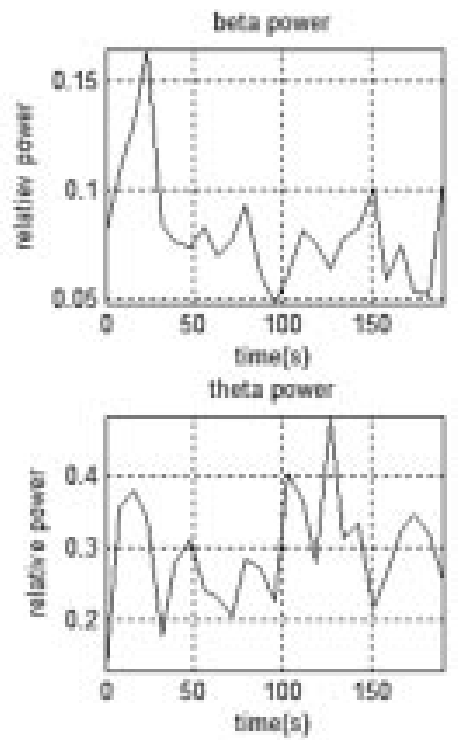

Fig 11. Relative power vs time plot 


\section{FEATURE CLASSIFICATION}

For proper classification and its evaluation, the features extracted from EEG and MEG data sets are segregated into training and testing sets. If the classification after comparing all the features for one segment $(5 s)$ resulted in sleep state, then the decision for that particular interval was given as "Sleep". If the classification indicated an "Awake" state, the decision was given as "Awake". Accordingly, binary values were allotted for the above two states. Classification into Sleep, awake and drowsy states are further determined using three different methods and their results are compared.

\section{A. Mean Comparison Test (MCT)}

The method of MCT (inspired by Antoine Picot, Sylvie Charbonnier and Alice Caplier) [7] is applied on the relative powers in the alpha band and beta band.
The features corresponding to a moving window of 5 seconds are compared with those corresponding to a fixed initial reference window of 20 s as shown on the figure 11. The MCT output is shown in fig. 12. The average value of relative alpha power of the moving window is compared to that of the fixed window. The value of relative power calculated from the fixed window sets the threshold for classification. The classical MCT has a limited application due to the fact that the theoretical variance values are unknown. The main advantage of the MCT is that it can be implemented online. If the normalized value of relative power is greater than the threshold (for relative alpha power), or less than threshold (for relative beta power), then the condition is considered to be drowsy; otherwise the condition is considered to be awake. This method does not need any training and testing data

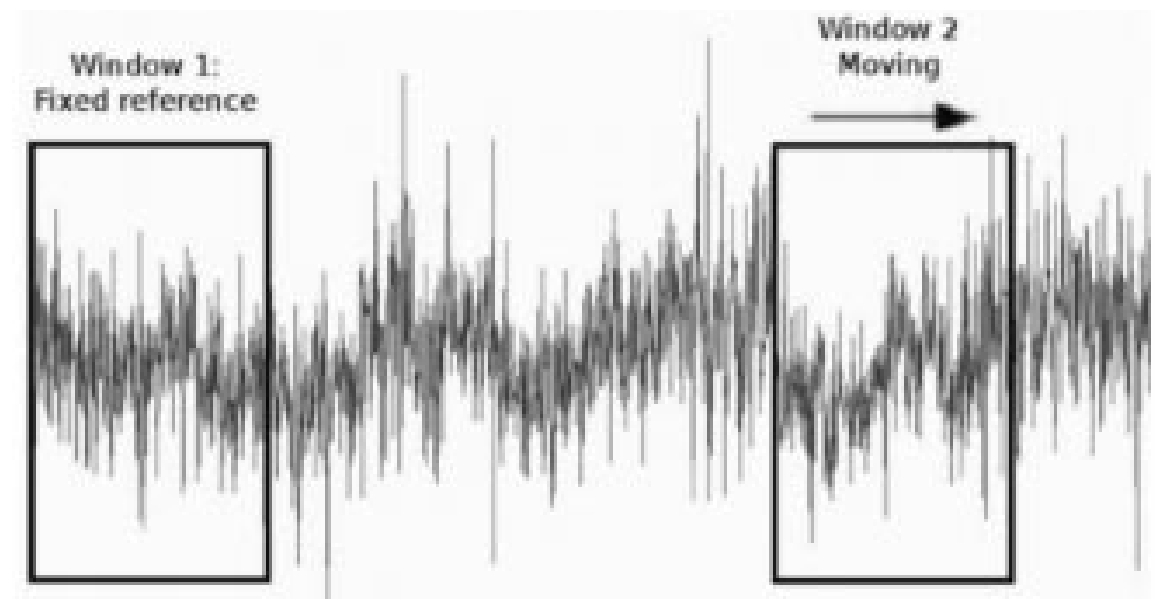

Fig 12. Illustration of the Windows for MCT (window size $5 \mathrm{~s}$ )

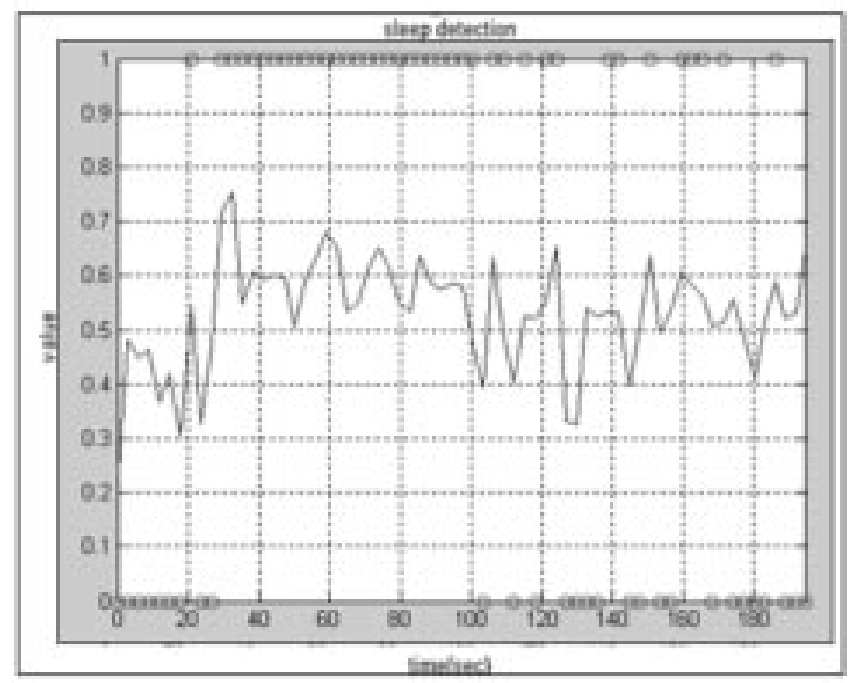

Fig. 13: MCT output 
sets. The threshold is set automatically from the fixed window's average relative power.

\section{Results And Discussions}

The feature extraction and its efficient usage by a classifier determine the performance and accuracy of the classifier. For best results many training and testing inputs should be given to the classifier, to increase sensitivity and reduce misclassifications.

\section{A. Performance and results of MCT}

MCT was applied to the feature relative alpha power, and following results were obtained:

Table 5.1: Performance comparison of MCT

\begin{tabular}{|c|c|c|c|}
\hline Signal & Sensitivity (\%) & Specificity (\%) & Accuracy (\%) \\
\hline EEG & 86.2745 & 50.00 & 77.6119 \\
\hline MEG & 82.4456 & 60.4453 & 74.6543 \\
\hline
\end{tabular}

\section{CONCLUSION}

From the above results of MCT, it can be concluded that if the normalized value of relative power is greater than the threshold (for relative alpha power), or less than threshold (for relative beta power), then the condition is considered to be drowsy; otherwise the condition is considered to be awake. Also, when compared to EEG, MEG results show better accuracy. Since, EEG is versatile and a cheaper method when compared to MEG, it can be implemented online for real time applications, and MEG can be used for source localization in sleep related studies due to its better spatial resolution.

\section{REFERENCES}

[1] M.P. Janawadkar, R. Baskaran, Rita Saha, K.Gireesan, R. Nagendran, L.S. Vaidhyanathan, J. Jayapandian and T.S. Radhakrishnan (1999), 'Squids: Highly Sensitive Magnetic Sensors', Current Science, Vol.77, No.6, Pg: 759-769.

[2] Cohen.D, Cuffin, B.N. (1983), 'Demonstration of useful Differences between the Magnetoencephalogram and Electroencephalogram', Electroencephalograph Clinical Neurophysiology, Vol. 56, Pg: 38-51.

[3] Barth.D.S, Sutherling.W.W, Beatty.J (1986), Intracellular Currents Of Interictal Penicillin Spikes: Evidence From Neuromagnetic Mapping',Brain Res Dev, Vol.368, Pg: 36-48.
[4] Mahalanobis.P.C (1936), 'On The Generalized Distance In Statistics' Proceedings Of The National Institute Of Sciences Of India, Vol 2 (1), Pg : 49-55.

[5] A.Fort, R.Martin, S.Dalgault, C.Delpuech, And A.Jacquet Andrieu, (2010), 'Attention And Processing Of Relevant Visual Information While Simulated Driving: A Meg Study', Ifmbe Proceedings, Vol.28, Pg: 322-325.

[6] Elisa Magosso, Mauro Ursino, Federica Provini, Pasquale Montagna,(2007), 'Wavelet Analysis Of Electroencephalographic And Electro-Oculographic Changes During The Sleep Onset Period', Proceedings Of The 29th Annual International Conference Of The leee Embs, Vol. 07, Pg: 4006-4010.

[7] Antoine Picot, Sylvie Charbonnier And Alice Caplier (2008), 'On-Line Automatic Detection Of Driver Drowsiness Using A Single Electroencephalographic Channel', 30th Annual International Conference Of The leee Engineering In Medicine And Biology Society, Embc'08, lembs, Vol.10, Pg: 1-20.

[8] Muhammed B. Kurt, Necmettin Sezgin, Mehmet Akin, Gokhan Kirbas, Muhittin Bayram (2009), 'The Ann-Based Computing Of Drowsy Level', Expert Systems With Applications (Elsevier), Vol: 36, Pg: 2534-2542.

[9] Inan Guler, Elif Derya Ubeyli, (2005), 'Adaptive Neuro Fuzzy Inference System For Classification Of Eeg Signals Using Wavelet Coefficients', Journal Of Neuroscience Methods (Elsevier), Pg: 1-9.

[10] Nikhil R. Pal, Chien-Yao Chuang, Li-Wei Ko, Chih-Feng Chao, Tzyy- Ping Jung, Sheng-Fu Liang, And Chin-Teng Lin, (2008),' Eeg-Based Subject- And Session-Independent Drowsiness Detection: An Unsupervised Approach', Journal On Advances In Signal Processing, Volume 2008, Article Id 519480, Pg: $1-11$.

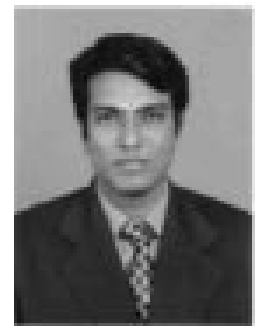

Mr. R. Sivarama Krishnan graduated in the discipline of Electronics and Communication Engineering from Madurai Kamaraj University and completed his Post Graduation in Medical Electronics from Anna University. He has more than nine years of teaching experience to his credit in diverse areas of electronics and communication and medical electronics. $\mathrm{He}$ is currently pursuing $\mathrm{PhD}$ under Information and communication engineering discipline at Anna University of Technology, Chennai 